\title{
Spatial and spatio-temporal analysis of malaria cases in Zimbabwe
}

\author{
Isaiah Gwitira ${ }^{1 *}$ D, Munashe Mukonoweshuro' , Grace Mapako ${ }^{1}$, Munyaradzi D. Shekede ${ }^{1}$, Joconiah Chirenda² \\ and Joseph Mberikunashe ${ }^{3}$
}

\begin{abstract}
Background: Although effective treatment for malaria is now available, approximately half of the global population remain at risk of the disease particularly in developing countries. To design effective malaria control strategies there is need to understand the pattern of malaria heterogeneity in an area. Therefore, the main objective of this study was to explore the spatial and spatio-temporal pattern of malaria cases in Zimbabwe based on malaria data aggregated at district level from 2011 to 2016.
\end{abstract}

Methods: Geographical information system (GIS) and spatial scan statistic were applied on passive malaria data collected from health facilities and aggregated at district level to detect existence of spatial clusters. The global Moran's I test was used to infer the presence of spatial autocorrelation while the purely spatial retrospective analyses were performed to detect the spatial clusters of malaria cases with high rates based on the discrete Poisson model. Furthermore, space-time clusters with high rates were detected through the retrospective space-time analysis based on the discrete Poisson model.

Results: Results showed that there is significant positive spatial autocorrelation in malaria cases in the study area. In addition, malaria exhibits spatial heterogeneity as evidenced by the existence of statistically significant $(P<0.05)$ spatial and space-time clusters of malaria in specific geographic regions. The detected primary clusters persisted in the eastern region of the study area over the six year study period while the temporal pattern of malaria reflected the seasonality of the disease where clusters were detected within particular months of the year.

Conclusions: Geographic regions characterised by clusters of high rates were identified as malaria high risk areas. The results of this study could be useful in prioritizing resource allocation in high-risk areas for malaria control and elimination particularly in resource limited settings such as Zimbabwe. The results of this study are also useful to guide further investigation into the possible determinants of persistence of high clusters of malaria cases in particular geographic regions which is useful in reducing malaria burden in such areas.

Keywords: Malaria, GIS, SaTscan, Spatial pattern, Spatial heterogeneity, Cluster analysis, Zimbabwe

*Correspondence: gwitsakuely@gmail.com; gwitirai@gis.uz.ac.zw ${ }^{1}$ Department of Geography Geospatial Sciences and Earth Observation, University of Zimbabwe, P. O. Box MP 167, Mount Pleasant, Harare, Zimbabwe

Full list of author information is available at the end of the article

\section{Background}

Compared with other human diseases, malaria remains one of the most serious public health problem associated with high morbidity and mortality in most developing countries [1-3]. In 2018 alone, 228 million malaria cases and 405000 deaths were recorded worldwide with the World Health Organisation (WHO) African Region contributing $93 \%$ of the cases and $94 \%$ of the deaths [4]. Although malaria has been successfully eradicated in

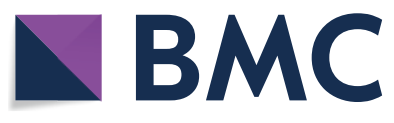

(c) The Author(s) 2020. Open Access This article is licensed under a Creative Commons Attribution 4.0 International License, which permits use, sharing, adaptation, distribution and reproduction in any medium or format, as long as you give appropriate credit to the original author(s) and the source, provide a link to the Creative Commons licence, and indicate if changes were made. The images or other third party material in this article are included in the article's Creative Commons licence, unless indicated otherwise in a credit line to the material. If material is not included in the article's Creative Commons licence and your intended use is not permitted by statutory regulation or exceeds the permitted use, you will need to obtain permission directly from the copyright holder. To view a copy of this licence, visit http://creativecommons.org/licenses/by/4.0/. The Creative Commons Public Domain Dedication waiver (http://creativeco mmons.org/publicdomain/zero/1.0/) applies to the data made available in this article, unless otherwise stated in a credit line to the data. 
high income and most middle income countries, the disease remains a major health problem and is a top killer infectious disease in low income countries [5]. In most parts of Zimbabwe, Plasmodium falciparum is the most common and efficient malaria parasite that accounted for $99.7 \%$ of the estimated cases in 2018 [4] while P. ovale and $P$. malariae account for the remainder. The primary vector mosquito species responsible for most malaria transmission in Zimbabwe are Anopheles arabiensis and Anopheles funestus sensu stricto [6,7].

In the past few years, malaria incidence and mortality have declined significantly across the globe $[8,9]$. For instance, mortality rate decreased by $62 \%$ while malaria incidence decreased by $41 \%$ between 2000 and 2015 [10, 11]. The decline in malaria incidence and mortality is mainly attributed to malaria control interventions such as indoor residual spraying (IRS) and use of insecticide treated nets $[10,12]$. Zimbabwe experienced a substantial decline in malaria cases of up to $81 \%$ from 2003 to 2015 across all age groups [13]. As a result of the substantial decline in malaria cases in Zimbabwe, the country adopted the global and regional agenda for malaria elimination by 2030 [14]. The target for the country is to reduce malaria incidence to $5 / 1000$ by end of 2020 [15]. As malaria transmission continue to decline, prevention and control interventions will increasingly rely on accurate knowledge of the spatial distribution of high-risk geographic areas to support malaria elimination. This could be useful in optimal allocation of limited resources to ensure that areas with the highest malaria burden are given priority [16, 17]. Despite the declining burden of malaria, there still exist periodic outbreaks of malaria which exhibit spatial heterogeneity across different regions through time and space. Mapping malaria spatial heterogeneity is important to better understand transmission dynamics $[18,19]$.

The spatial heterogeneity in malaria transmission has resulted in malaria occurring in transmission clusters $[19,20]$. The spatial heterogeneity in malaria is largely attributed to variation in environmental risk factors at the macro (e.g., temperature, precipitation) and the micro (e.g., local elevation, land use) spatial scales [21]. In this case, a malaria cluster is an area characterised by unusually high number of cases than expected within a population at a particular place at a given time [22]. As malaria occurrence exhibit spatial heterogeneity, strategies aimed at reducing or controlling the disease hinge upon objective and accurate characterisation of its clusters as a first step towards identifying areas with elevated malaria risk for prioritisation of interventions [18, 19]. Evidence from previous studies suggest that targeting malaria control interventions at high risk areas is cost effective and is bound to increase equity compared with undirected control [23]. Such targeted interventions ultimately reduce malaria mortality and morbidity [24]. Focussing malaria control in high risk areas is recommended by WHO in both elimination and post elimination settings [25].

Although previous studies assessed the spatial and temporal variation in malaria occurrence, most of these studies lacked the appropriate spatial scale that enables optimal planning at the national level [26-29]. In addition, most of the studies were either based on longitudinal cohort studies or limited in temporal duration [2, 30]. For example, some studies observed clustering of malaria cases at micro-geographic scale such as ward level in Gwanda district of Zimbabwe [31]. Similarly, it was also established that malaria exhibited spatio-temporal clusters at village level in China [29]. In addition, high malaria risk areas were identified in Hubei Province of China based on scan statistics [32]. In another study, it was found that malaria case distribution is characterised by spatial, temporal and spatiotemporal heterogeneity in unstable transmission areas in North-west Ethiopia [30]. Although these studies provide useful insights in understanding the spatial and temporal pattern of malaria, knowledge on the spatial and temporal pattern of malaria at a level where malaria interventions are commonly planned remain patchy. This is despite the fact that spatial analysis becomes much more meaningful when the spatial unit at which analysis is performed is representative of the expected epidemiological dynamics [21]. This will then mean the resulting national-level maps from such analysis will be justifiably utilized to prioritize high risk areas [21].

Despite the fact that effective malaria intervention warrants understanding of malaria heterogeneity at larger spatial scales for the purposes of resource allocation before focussing on microgeographic regions, studies at this scale remain largely limited. To fill this gap, this study utilised relatively long term malaria case data at district level, that is, the spatial epidemiological administrative unit at which malaria interventions and control are planned to determine not only persistent and stable clusters but emerging clusters as well [33]. The determination of spatial pattern of malaria at district level is also important in understanding possible interactions among neighbouring districts which is fundamental during malaria elimination.

To determine the spatial pattern of malaria clustering, it is important to adopt or even develop methods that can reliably and accurately detect malaria clusters in space and time. To date, several methods have been used to detect spatial and space-time clusters and these include, ClusterSeer [34], GeoSurveillance [35], kernel density [36], SaTScan [37] and Flex Scan [38]. The 
choice of a cluster detection technique can be guided by its sensitivity and specificity in addition to the power to detect clusters [39, 40]. In this study, SaTscan was applied since results from previous studies indicated that it has the highest overall sensitivity compared to other methods such as Local Indicators of Spatial Autocorrelation (LISA) and Getis [39] hence its ability to detect true clusters. Moreso, the technique maintains reasonably high power for detecting clusters compared to methods such as LISA which are influenced by neighbours [41]. Techniques such as Getis-Ord $G_{i}{ }^{*}$ statistic suffer from multiple testing which is inherently accounted for in SaTscan [42]. In this way, SaTscan combines exploratory and confirmatory capabilities which enable explicit statistical assessment of spatial pattern across the landscape [39].

In this study, the main objective was to test whether there is statistically significant spatial and space-time clustering of malaria at district level in Zimbabwe. The main hypothesis was that malaria tends to occur in clusters and that these clusters have both spatial and temporal characteristics.

\section{Methods}

\section{Study area}

The study was carried out in Zimbabwe located in southern Africa between latitudes $15^{\circ} 30^{\prime \prime}$ and $22^{\circ} 30^{\prime \prime} \mathrm{S}$ and longitudes $25^{\circ} 00^{\prime \prime}$ and $33^{\circ} 10^{\prime \prime} \mathrm{E}$ (Fig. 1). The altitude of the study area ranges from 300 to $2590 \mathrm{~m}$ above mean sea level. Mean annual rainfall ranges from below $400 \mathrm{~mm}$ in the southern and north-western parts to over $1000 \mathrm{~mm}$ in the eastern and central parts of the country. The country records its lowest minimum temperatures in June or July while the maximum temperatures occur in October. Mean monthly temperatures vary from $15{ }^{\circ} \mathrm{C}$ in July to $24{ }^{\circ} \mathrm{C}$ in November, while the mean annual temperature varies from $18{ }^{\circ} \mathrm{C}$ in the high-altitude areas to $23{ }^{\circ} \mathrm{C}$ in the low altitude areas. The study area is characterised by a subtropical climate with three recognisable seasons which are the hot wet season or summer stretching from mid-November to March; the cold dry season or winter stretching from April to July; and the hot dry season or spring from August to mid-November [43]. Zimbabwe experiences seasonal and spatial variation in malaria transmission that is related to the country's climate especially rainfall pattern $[13,44]$. The malaria peak transmission season in Zimbabwe is between February and April [13]. According WHO, there were close to 14 million people in the country, and four million were at risk of malaria with close to 1 million confirmed cases in 2018. The country is landlocked and shares the border with Mozambique, Botswana, Zambia and South Africa (Fig. 1).

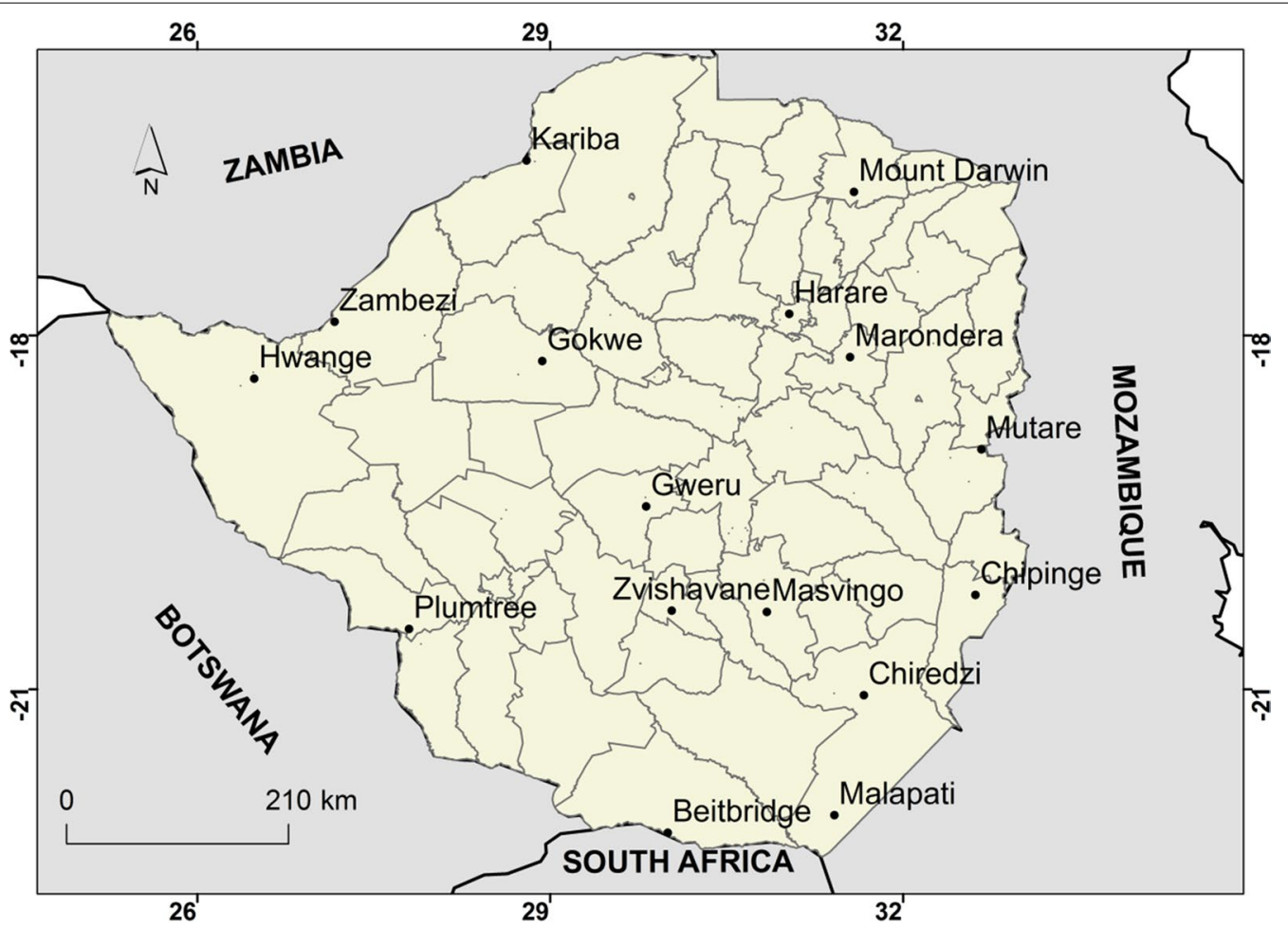

Fig. 1 Location of the study area 


\section{Data sources}

\section{Malaria case data}

The positive malaria cases recorded from 2011 to 2016 were obtained from geocoded health facilities in Zimbabwe and aggregated by month and year at district level. The country has over 1780 health facilities strategically placed within a $10-\mathrm{km}$ radius in villages and urban areas [13]. The health facilities are ranked into central, provincial, and district hospitals, as well as clinics/rural health centres (RHCs). Data on malaria incidence at district level from 2011 to 2016 were collected based on the annual submission from health centres to DHIS 2 . The data includes province, district, and centre name, date of diagnosis, age and gender. The data is for cases based on diagnostics using either rapid diagnostic tests (RDTs) or microscopy reported in each year [13]. Zimbabwe, like most developing countries in sub-Saharan Africa, adopted the District Health Information System (DHIS2) in 2010 to harmonise health data management [45]. The malaria cases at district level were plotted using ArcGIS 10.3 (ArcGIS Desktop: Release 10. Redlands, CA, USA) [46] by month and year to assess the distribution of malaria cases in the country.

For the Ministry of Health and Child Care, two main sources are used to feed the national surveillance system with routine malaria data i.e., the Health Management Information System (HMIS) and the Rapid Disease Notification System (RDNS) [47]. The RDNS, weekly short message service (SMS) is used to report malaria cases for approximately $95 \%$ of the health facilities. In addition, the HMIS obtains its data from monthly aggregated malaria cases and deaths from all health facilities [47]. In most developing countries in sub-Saharan Africa including Zimbabwe, routine health information systems are weak and there are widespread concerns about the quality and utility of malaria data generated from these systems [48, 49]. Despite concerns about data quality, Zimbabwe has made great strides on this aspect through government initiatives and international support. To improve data quality, the government adopted the Global Technical Strategy for Malaria 2016-2030 which stresses the need for adequate investment in the management and use of data from routine health information systems to support programme planning, implementation and evaluation [50].

\section{Population data}

Population data used in this study were obtained from the Zimbabwe National Statistical Agency (ZimStat) based on the 2012 National population census [51]. The 2010 and 2011 population data was based on a $1.1 \%$ projected growth rate from the 2002 National census [52] while the
2012 data was based on the 2012 National Census. The population for intercensal years for example 2013 to 2015 were determined using the projected annual growth rate of $1.2 \%$ based on the 2012 national census [53]. The population of intercensal years is based on projected growth rates because the country conducts a population census after every 10 years.

\section{Statistical data analysis \\ Testing for spatial autocorrelation}

Moran's $I$ [54], a global autocorrelation statistic was used to detect spatial pattern of malaria in the country. Using this technique, significant positive spatial autocorrelation of malaria cases imply that the distribution of malaria cases is more spatially aggregated than a random underlying spatial process.

The Moran's Index takes the form;

$$
I=\frac{n}{S_{o}} \frac{\sum_{i=1}^{n} \sum_{j=1}^{n} w_{i, j} z_{i} z_{j}}{\sum_{i=1}^{n} z_{i}^{2}}
$$

where $\mathrm{Z} i$ is the deviation of an attribute for feature $i$ from its mean $\left(\mathrm{x}_{\mathrm{i}}-\bar{X}\right), \mathrm{w}_{\mathrm{i}, \mathrm{j}}$ is the spatial weight between feature $i$ and $j, n$ is equal to the total number of features and $S_{o}$ is the aggregate of all spatial weights.

Moran's index ranges from -1 to +1 with a score of zero indicating the null hypothesis of no clustering. A positive score indicates clustering of malaria cases while a negative value shows that neighbouring areas are characterised by dissimilar malaria cases [55]. To perform spatial autocorrelation, the Queen Contiguity method was applied to define a weight matrix specifying the spatial relationships among the districts of Zimbabwe. This method was adopted since malaria is not directionally restricted and the districts are highly irregular in shape and size [56]. The significance of Moran's $I$ was assessed by employing Monte Carlo randomization where a statistically significant $(Z$ score $>1.96)$ indicated that neighbouring districts have similar malaria cases at county level.

\section{Detecting malaria clusters using SaTScan}

In this study, scan statistics [42] was applied in SaTScan v9.6 (https://www.satscan.org/) software to detect high cluster rate of malaria. In this case, spatial scan statistic, based on the discrete Poisson model, was applied to identify purely spatial clusters of malaria cases by year. On the other hand, the space-time scan statistic, based on Space-Time Poisson model was adopted to determine the presence of space-time clusters of malaria cases by month over the study period. Three datasets were prepared for use in SaTScan and these were: a case file representing annual malaria cases per 
each district $(n=59)$ from 2011 to 2016; a coordinate file representing geographic coordinates of the centroid of each district; and a population file representing the projected total population for each year from 2011 to 2016 for the respective district.

The program identified statistically significant retrospective clusters based on annual malaria cases aggregated per district in Zimbabwe from 2011 to 2016. SaTscan tests whether the number of malaria cases within any spatial window exceeds the number expected by a random process [57]. To achieve this, the centroid of each district was first determined and extracted in a GIS environment. The spatial join function in a GIS was then used to link the annual malaria cases for each year to the centroid of the districts.

Next, the annual malaria cases per district were converted to SaTscan format for use in the detection and analysis of clusters. For determining clusters, a cylindrical window with a circular geographic base centred on each district centroid and with height corresponding to time was applied [57]. The default value of $50 \%$ of the population at risk was adopted as recommended in literature $[37,58,59]$. Thus, clusters with statistical significance of $P<0.05$ were classified as significant clusters. As previously mentioned, the space-time clusters of malaria with high rates were detected using the retrospective space-time analysis based on the discrete Poisson model. To do this, data was arranged at a monthly scale from 2011 to 2016 and hence the time aggregation length was set to one month in SaTscan software. The space-time scan statistic was defined by a cylindrical window with a circular geographic base and whose height corresponded to a time interval i.e., a month in this case. The space-time analysis was applied to detect the seasonal pattern in malaria in the country. This technique is more robust as it combines exploratory and confirmatory capabilities which enable explicit statistical assessment of spatial patterns across the landscape [39] compared to Getis-Ord $\mathrm{G}_{i}{ }^{*}$.To detect significant space-time clusters, 999 Monte Carlo replications were performed under the null hypothesis of random distribution of malaria cases [32]. In this case, statistical significance was tested using a Poisson generalized log likelihood ratio test based on Monte-Carlo inference [32, 60].

The relative risk was calculated by comparing the observed number of cases of malaria within each window to the expected number, using a Poisson model. The most likely cluster (hereinafter, primary cluster) was identified based on the maximum log likelihood ratio [61]. In addition, other clusters with statistically significant log likelihood values were defined as secondary clusters. The criterion of no geographical overlap was used to report secondary clusters.

\section{Cluster frequency analysis}

To understand the persistence or emergence of potential malaria hotspots cluster frequency analysis was performed in a GIS environment. Specifically, the cluster frequency analysis was performed through counting the number of times a district was detected as part of a cluster using overlay analysis. This procedure yielded the number of times a district coincided with detected clusters whether primary or secondary.

\section{Results \\ Variation in monthly malaria incidence}

Figure 2 illustrates the variation in average monthly malaria incidence from 2011 to 2016 in Zimbabwe. From 2011 to 2016, a total of 1877794 malaria cases were recorded throughout the country. It can be observed that malaria incidence start to increase from December and reach the highest peak in February. In contrast, the lowest incidence is recorded during the dry months such as August and cold month such as July.

\section{Annual incidence of malaria}

An analysis of annual malaria cases shows that over the 6 years, the northern, north-eastern, eastern and southeastern districts of the country were characterised by high malaria incidence (Fig. 3). In contrast, the western, central and south western regions had low malaria incidence during the same period. In fact, more districts in the eastern districts of the country experienced high malaria incidence in any other year compared with other districts where malaria occurs.

\section{Spatial autocorrelation of malaria cases}

Spatial autocorrelation results based on the annual malaria cases showed that there was significant overall spatial autocorrelation in Zimbabwe across all the study years (Table 1). The results demonstrate that malaria cases highly cluster at country level for all the years under study.

\section{Spatial clusters of malaria from 2011 to 2016}

The results for the statistically significant $(P<0.05)$ primary and secondary spatial clusters as well as the corresponding relative risk for high rates of malaria occurrence identified by purely spatial scan statistic based on the discrete Poisson model are illustrated in Fig. 4. The results illustrate that there was significant spatial clustering of malaria cases in specific districts from the years 2011 to 2016 (Fig. 4, Table 2). Over the six years, primary clusters of malaria were concentrated in the eastern region of the country. The number 


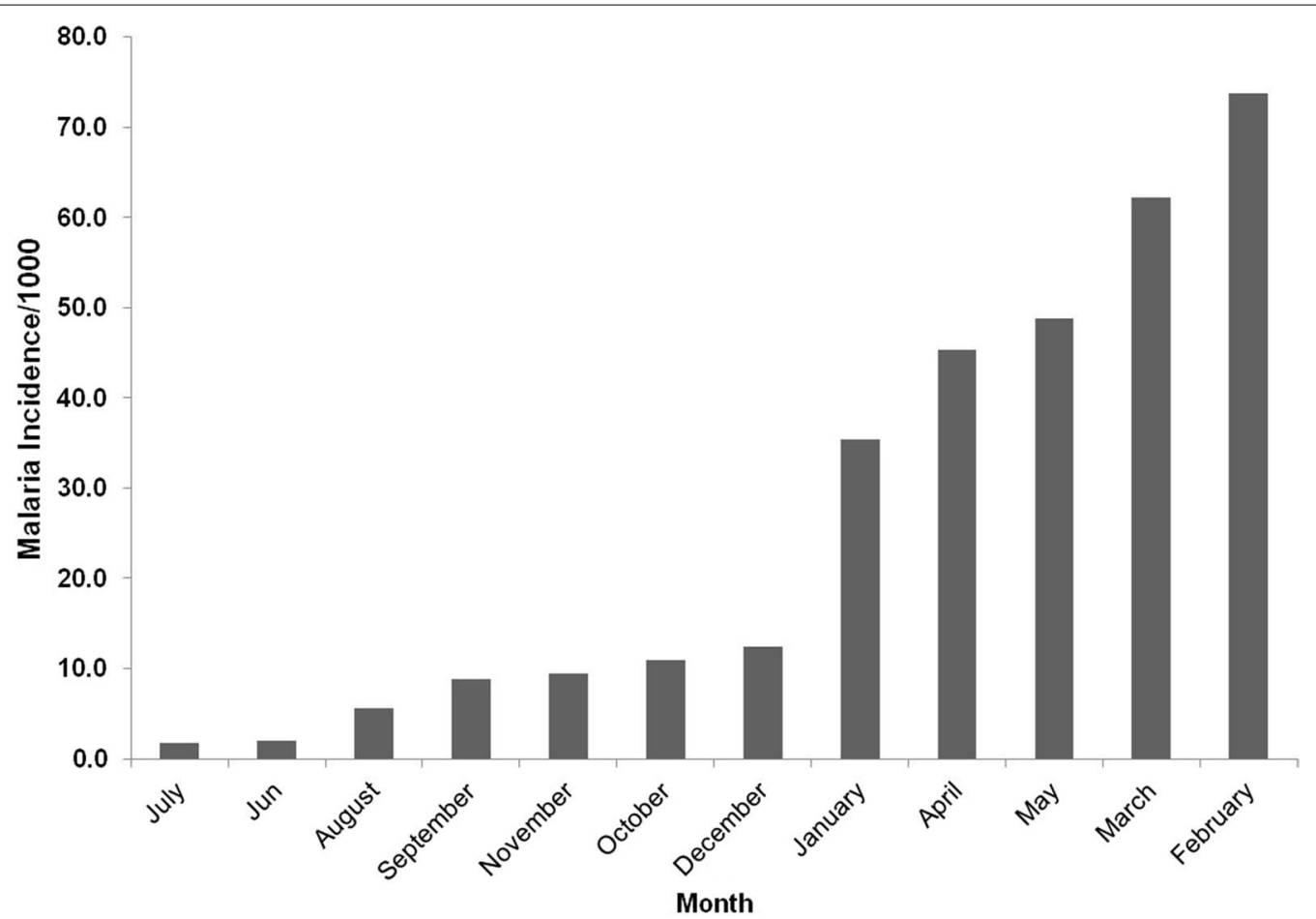

Fig. 2 Average monthly malaria incidence from 2011 to 2016

of districts covered by the primary clusters increased from four in 2011 to 12 in 2016. In general, secondary clusters are characteristic of the eastern, northern, south-western and south-eastern regions of the country. However, the spatial location and size of these secondary clusters varied by year (Fig. 4).

Further, results illustrate that the lowest number of malaria cases within a cluster was 5414 (2011) while the highest was 92324 (2012). Across all the years under study, the most likely clusters had higher than expected malaria cases (Table 2).

\section{Frequency of cluster occurrence from 2011 to 2016}

The frequency of occurrence of malaria clusters within districts based on scan statistics is illustrated in Fig. 5. It is observed that districts in the northern, north-eastern, eastern and south-eastern regions of the country had the highest frequency of malaria clusters. Districts at the margins of high malaria cluster districts had low frequency of cluster occurrence. In contrast the central, western and south western districts had no malaria clusters during the period under consideration.

\section{Space-time clusters of malaria}

Results of space-time Poisson model show four spatialtemporal malaria clusters that were detected from 2011 to 2016 (Fig. 6, Table 3). The four statistically significant spatio-temporal clusters consisted of one primary cluster and three secondary clusters. The primary cluster was located in the north eastern region and covers eight administrative districts (Fig. 6).

The primary cluster was detected from the 1 st of December 2012 to the 31st of May 2014. The primary cluster persisted in this region for three seasons and covered eight districts (Table 3 ).

\section{Discussion}

In this study, Geographic Information System coupled with a spatial scan statistical method were successfully applied to explore spatial and temporal patterns of malaria clusters between 2011 and 2016 at district level in Zimbabwe. The results of this study showed significant global spatial autocorrelation of malaria cases from 2011 to 2016 which indicates that the spatial distribution of malaria followed a clustered pattern. The results confirm findings from previous studies that observed the tendency of malaria to cluster in particular geographic regions mostly derived by the spatial heterogeneity in the factors that drive transmission of the disease [20, 31]. Based on previous studies, the spatial heterogeneity in malaria is largely attributed to variation in environmental risk factors at the macro (e.g., temperature, precipitation) and the micro (e.g., local elevation, land use) spatial scales [21]. From the observed detected pattern, 


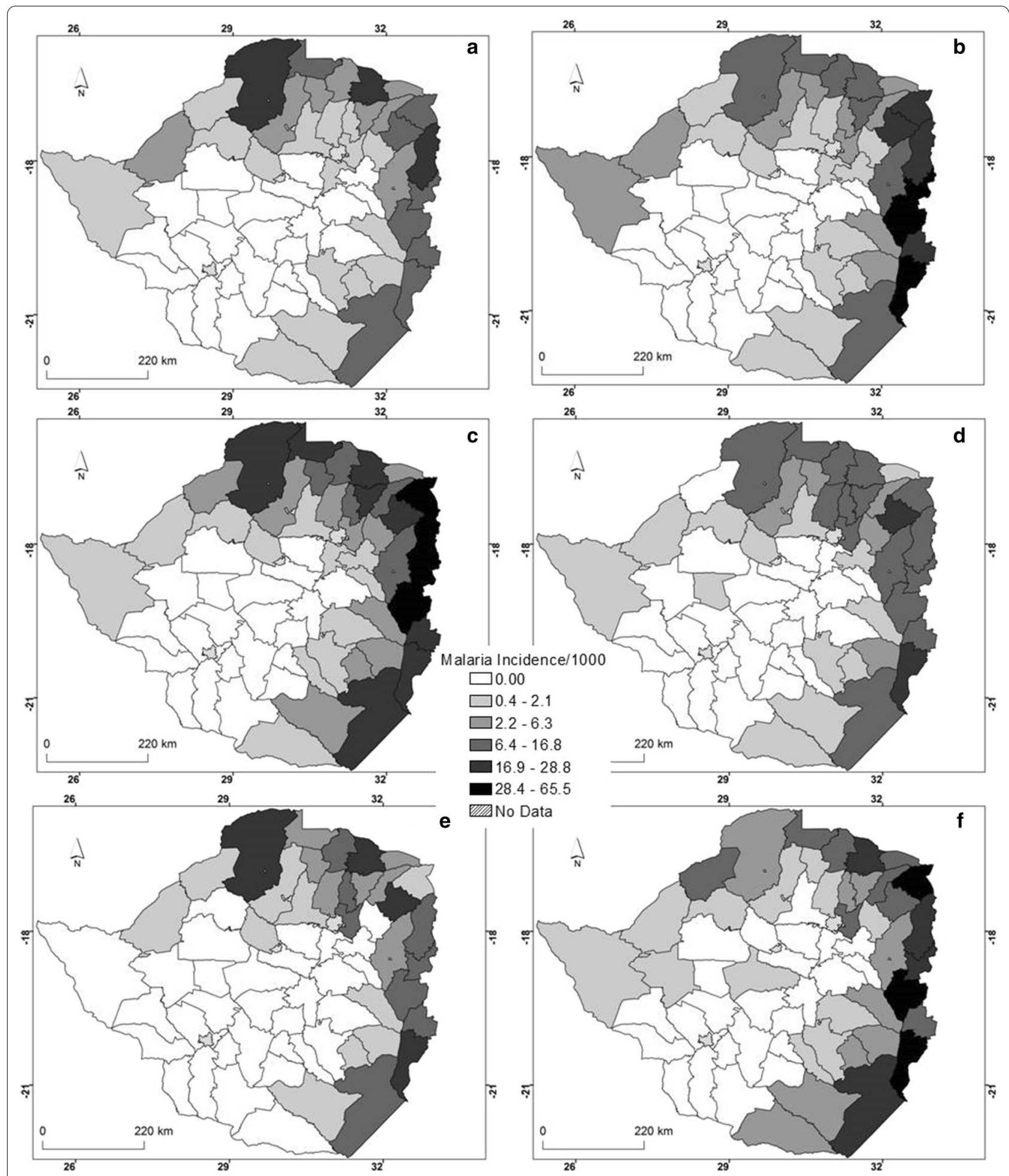

Fig. 3 Spatial distribution of annual malaria incidence for a 2011, b 2012, c 2013, d 2014, e 2015 and f 2016

the variation in malaria cases coincide with the distribution of the preferred habitat of the Anopheles mosquitoes which are the main vectors that transmit $P$. falciparum parasite [21]. Thus, our study further provides evidence of spatial heterogeneity in the occurrence of malaria in the affected regions. 
Table 1 Spatial autocorrelation test on malaria cases from 2011 to 2016

\begin{tabular}{llcl}
\hline Year & Moran's index & $\boldsymbol{Z}$ score & $\boldsymbol{P}$-value \\
\hline 2011 & 0.53 & 20.00 & 0.00 \\
2012 & 0.56 & 21.45 & 0.00 \\
2013 & 0.41 & 15.67 & 0.00 \\
2014 & 0.45 & 17.27 & 0.00 \\
2015 & 0.57 & 21.48 & 0.00 \\
2016 & 0.25 & 112.09 & 0.00 \\
\hline
\end{tabular}

Results of purely spatial analysis based on the poisson model showed that primary and secondary clusters of malaria persisted in the northern, north-eastern, eastern and south-eastern districts of Zimbabwe. The results show that consistently over the study period, malaria clusters occur in different sizes and at different locations. This is important in identifying stable cluster areas which persist in areas of high malaria burden [20]. The results support the hypothesis that malaria cases tend to significantly cluster within certain geographic units albeit with observable shifts over time. This may indicate that the occurrence of malaria in Zimbabwe is characterised by spatial heterogeneity as high-risk areas still exist particularly in the north north-east, east and south eastern districts of the country [31]. The high risk areas detected in this study are consistent with malaria hotspots detected through Getis $\mathrm{G}_{i}{ }^{*}$ statistic analysis and were closely related to high vector habitat suitability [2]. In addition, the high risk areas coincide with the high and perennial malaria risk zones delineated through malaria risk stratification in Zimbabwe [62]. As the country moves towards malaria elimination [13], there is need to prioritise control efforts by focussing on high risk areas as these are possible reservoirs of malaria transmission [63]. The detection of statistically significant malaria clusters is a critical step towards spatial targeting and selection of appropriate population level interventions as these clusters are potential reservoirs for future infection [20, 27]. Through the detection of clusters, affected countries can shift from malaria control to malaria elimination which is one of the key goals of the WHO Global Technical Strategy for Malaria 2016-2030 [14]. The targeting of high risk areas for malaria control aligns with the United Nations Sustainable Development Goal (SDG) number three which is seeks to promote good health and well-being through scaling up of malaria interventions $[4,64]$.

Results of space-time analysis showed that malaria clusters tend to occur in particular months e.g. December to May. The fact that most clusters were detected during this period is not surprising as the country receives most of its rainfall between December and March which dissipates towards April and May [13]. The high amount of rainfall coupled with relatively high temperatures during this period provides optimal conditions for mosquito breeding and subsequent malaria transmission [13]. Previous studies have shown that this period coincides with the malaria epidemic season in Zimbabwe and offers favourable climatic conditions in high risk months for malaria transmission [7, 13, 44]. When combined with location specific information on malaria clustering, results of space-time clustering further points to the importance of incorporating these two aspects in order to fully understand malaria transmission dynamics. Such insights would not have been generated had the study only adopted either purely spatial or purely temporal approach in modelling clustering of malaria. The results of space-time analysis can then be utilised to plan timing of control interventions by targeting those months where clusters are common. This would require deviation from the usual practice where indoor residual spraying is done well before the malaria season.

What makes this study different from other previous studies is that, unlike previous studies, this study used malaria case data for a relatively long period (six years) which provides important insights in the persistence of clusters (stable clusters) in certain geographic regions. In addition, this study integrated space and time in one analytical framework which provides new insights into the evolution of malaria not only in the spatial but also in the temporal domain. This study utilised one of the most robust methods of cluster detection to understand the pattern of malaria clusters unlike previous studies which have mostly utilised hotspot analysis techniques such the Getis $\mathrm{G}_{i}{ }^{*}$ statistic [2]. The technique used in this study has both high specificity and sensitivity hence provides a balance in terms of committing at type 1 or type 2 errors. Furthermore, the high risk areas identified in this study may serve as important starting points for future disease surveillance in resource limited environments such as Zimbabwe. Apart from providing disease surveillance targets, such high risk areas could be prioritised during resource allocation to achieve effective disease control. However, further research should be focussed in these areas to fully understand disease etiology and local factors that support elevated malaria risk.

Data quality related to use of retrospective data may have affected the results of this study. Most developing countries are characterised by incomplete reporting of routine data, non-reporting, missing data and poor data aggregation frameworks [65]. Nonetheless, malaria case-management and data quality have greatly improved 


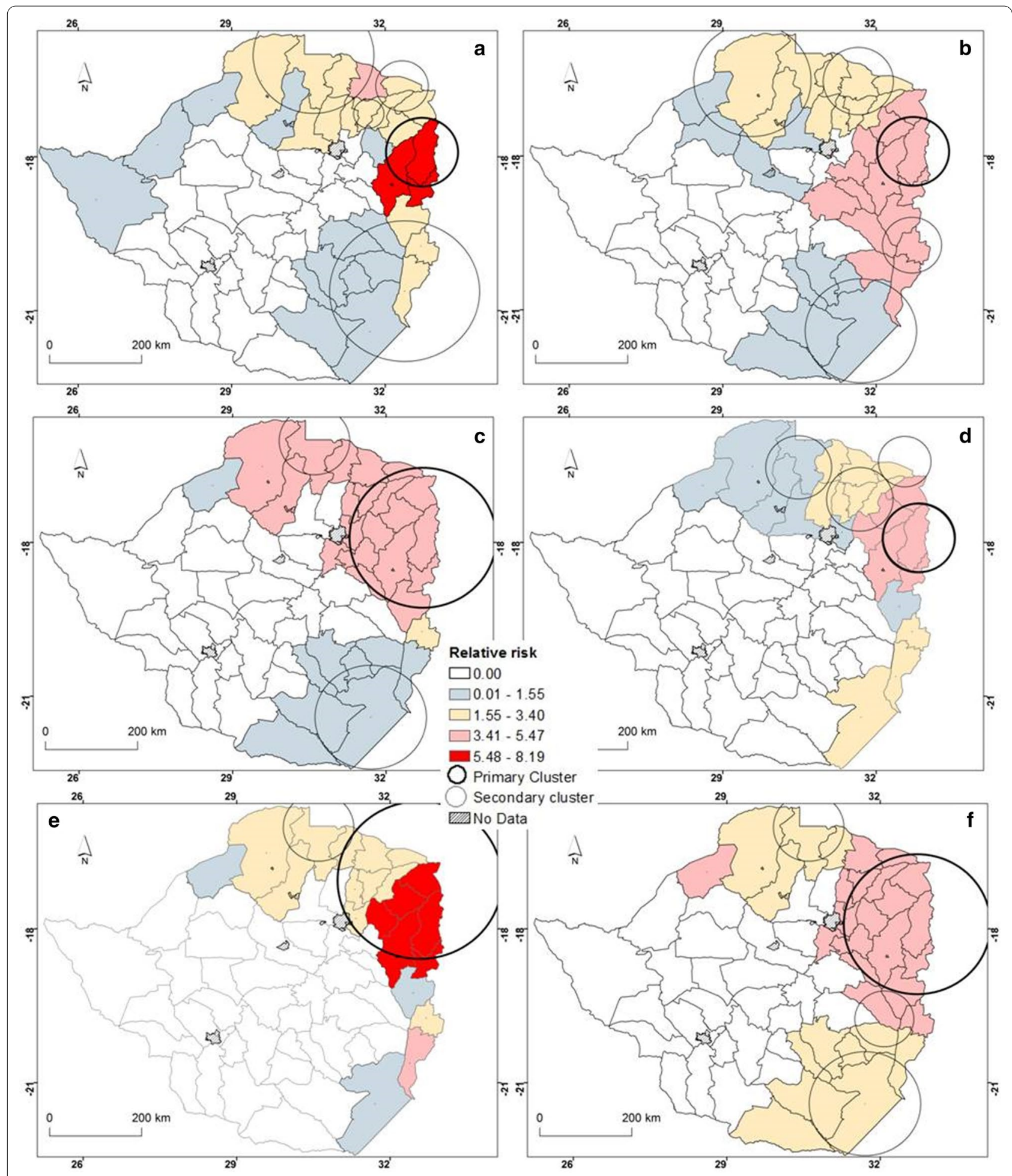

Fig. 4 Spatial distribution of malaria clusters detected by purely spatial for a 2011, b 2012, c 2013, d 2014, e 2015 and f 2016. (The primary cluster is illustrated by a darker outline) 
Table 2 Significant malaria clusters detected using the purely spatial clustering

\begin{tabular}{|c|c|c|c|c|c|c|c|c|}
\hline Year & Cluster type & $\begin{array}{l}\text { Cluster areas } \\
(n)\end{array}$ & Observed & Expected & RR & Radius(km) & LLR & $P$-value \\
\hline & A & 3 & 48569 & 9569 & 6.38 & 74 & 44198 & 0.00 \\
\hline & B & 6 & 58222 & 22305 & 3.27 & 126 & 23850 & 0.00 \\
\hline \multirow[t]{5}{*}{2011} & B & 2 & 13937 & 4520 & 3.24 & 55 & 6506 & 0.00 \\
\hline & B & 7 & 47530 & 36405 & 1.40 & 152 & 1937 & 0.00 \\
\hline & B & 2 & 5414 & 5015 & 1.08 & 32 & 16 & 0.00 \\
\hline & A & 3 & 92324 & 17148 & 6.94 & 74 & 89397 & 0.00 \\
\hline & B & 2 & 74241 & 22710 & 3.88 & 60 & 40668 & 0.00 \\
\hline \multirow[t]{4}{*}{2012} & B & 5 & 44030 & 25085 & 1.86 & 73 & 6386 & 0.00 \\
\hline & B & 3 & 42155 & 28805 & 1.53 & 113 & 2982 & 0.00 \\
\hline & B & 5 & 34236 & 31941 & 1.08 & 122 & 89 & 0.00 \\
\hline & A & 12 & 247572 & 108723 & 3.78 & 152 & 97033 & 0.00 \\
\hline \multirow[t]{4}{*}{2013} & B & 2 & 41339 & 10382 & 4.28 & 74 & 27258 & 0.00 \\
\hline & B & 3 & 52771 & 37524 & 1.46 & 113 & 3026 & 0.00 \\
\hline & A & 3 & 52868 & 12087 & 5.28 & 74 & 40965 & 0.00 \\
\hline & B & 4 & 36004 & 14553 & 2.72 & 69 & 12175 & 0.00 \\
\hline \multirow[t]{3}{*}{2014} & B & 2 & 16088 & 5243 & 3.21 & 55 & 7438 & 0.00 \\
\hline & B & 3 & 17509 & 14423 & 1.23 & 69 & 329 & 0.00 \\
\hline & A & 1 & 28145 & 5788 & 5.47 & 114 & 23439 & 0.00 \\
\hline \multirow[t]{3}{*}{2015} & B & 2 & 13261 & 5885 & 2.34 & 74 & 3533 & 0.00 \\
\hline & A & 12 & 211251 & 88565 & 3.88 & 152 & 88392 & 0.00 \\
\hline & B & 2 & 79698 & 23800 & 3.92 & 60 & 44713 & 0.00 \\
\hline \multirow[t]{2}{*}{2016} & B & 3 & 77507 & 30514 & 2.90 & 113 & 28321 & 0.00 \\
\hline & B & 2 & 31355 & 12709 & 2.59 & 74 & 10118 & 0.00 \\
\hline
\end{tabular}

$A$ primary cluster, $B$ secondary, $R R$ relative risk; $L L R$ log likelihood ratio

particularly parasitological testing as well as the adoption of electronic databases such as DHIS2. Although routine data from health facilities is known to underestimate malaria burden due to the above mentioned factors, the data is still useful in understanding the spatial distribution of malaria in endemic regions [66, 67]. Thus, the results of this study provide an important basis for planning and implementation of malaria control strategies.

Although spatial and spatio-temporal clusters of malaria were successfully detected using data from 2011 to 2016, one limitation is that the malaria cases used in this study did not differentiate local and imported cases. It is important to differentiate local and imported malaria cases particularly given the observation that most of the high rates of malaria clusters tend to be concentrated along borderline areas $[68,69]$. The challenge is that a greater part of the borders of the country are porous making it difficult to monitor movement. For example, to the east Zimbabwe shares a $730 \mathrm{~km}$ border with Mozambique which is also known to have high malaria cases while to the south-east the country borders with South Africa along the Limpopo valley (a malaria endemic region) [70-72] and to the North it borders with Zambia
[73, 74]. Imported cases could have influenced the size and location of high rates of malaria clusters detected in this study. Migration related malaria remains a major problem for Zimbabwe especially in the eastern parts of the country. The occurrence of malaria due to migration could be as a result of locals travelling to neighbouring Mozambique during the day and contracting malaria which is then reported in eastern districts. Additionally, populations from neighbouring country may access treatment in Zimbabwe's eastern districts where the treatment is free to patients. Usually these cases are not reported in the DHIS2 database despite receiving treatment. The use of genomic surveillance may address the first challenge of locals contracting malaria from the neighbouring country. Introducing a data point recording non-resident malaria patients would allow an accurate characterisation of the burden of malaria in the eastern border districts. There is therefore need for national data collection systems to incorporate imported cases in their systems. To achieve this, there is need for closer collaboration with neighbouring countries.

The information generated in this study could be important in strengthening cross border collaboration 


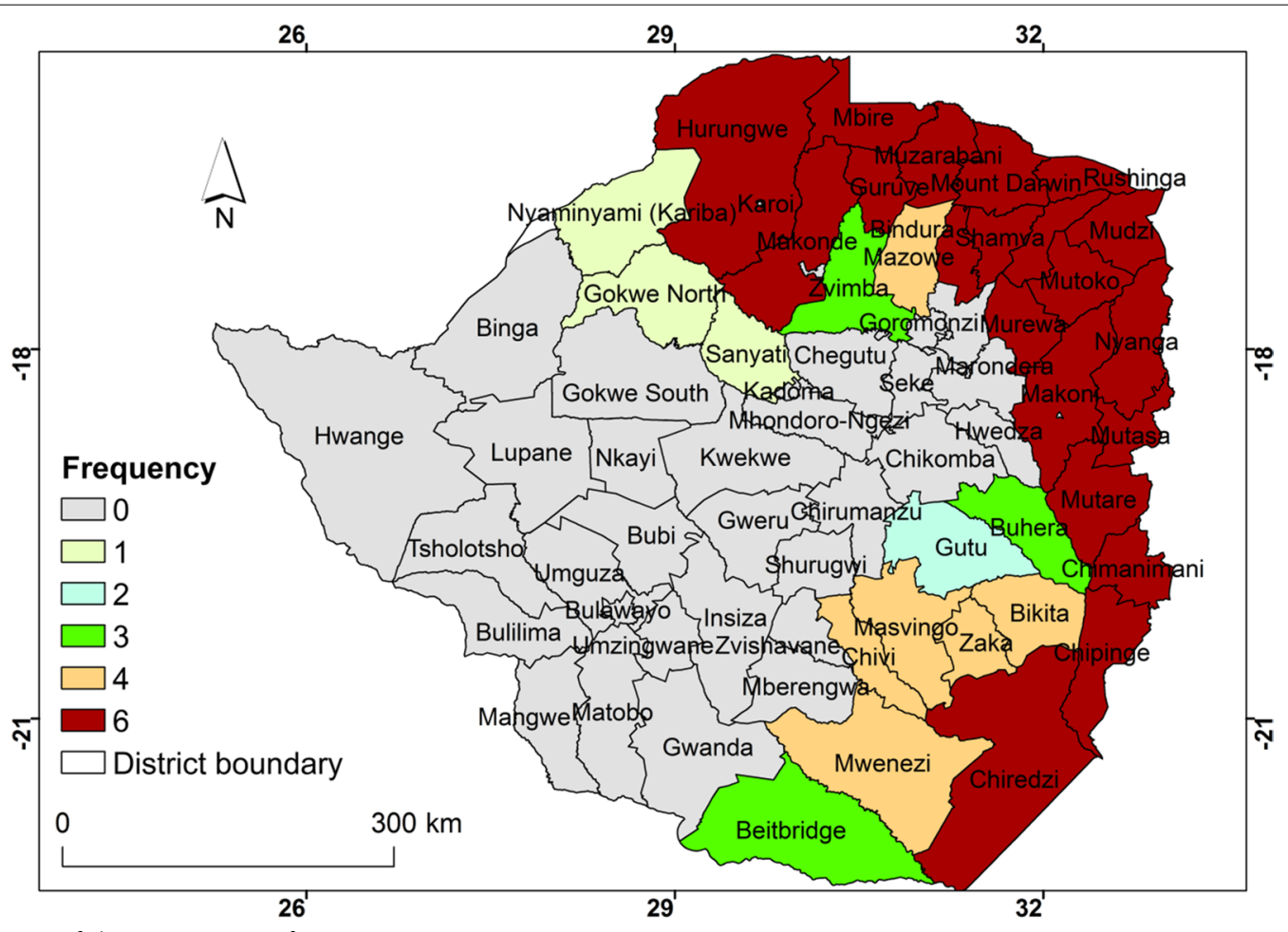

Fig. 5 Frequency of cluster occurrence from 2011 to 2016

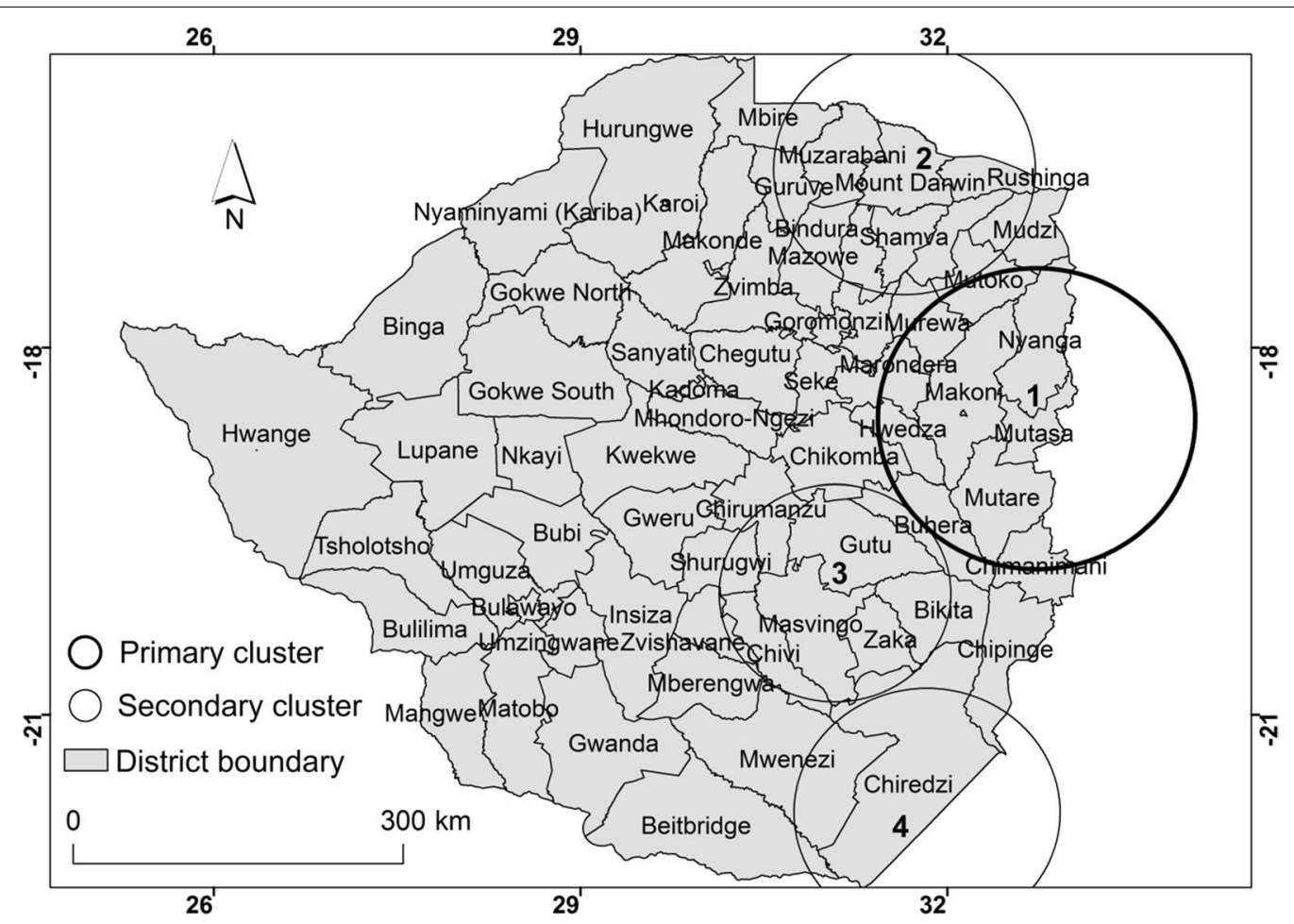

Fig. 6 Spatial distribution of detected space-time clusters of malaria from 2011 to 2016 
Table 3 Spatial-temporal high risk clusters of malaria cases detected using space-time Poisson model from 2011 to 2017

\begin{tabular}{llllllcc}
\hline Cluster \# & \# of Location & Start date & End date & LLR & P value & RR & Radius \\
\hline$* 1$ & 8 & $01 / 12 / 2012$ & $31 / 05 / 2014$ & 331605 & 0.001 & 6.10 & 137 \\
2 & 9 & $01 / 03 / 2014$ & $31 / 05 / 2014$ & 148263 & 0.001 & 10.89 & 114 \\
3 & 6 & $01 / 01 / 2016$ & $31 / 05 / 2016$ & 83074 & 0.001 & 7.74 & 113 \\
4 & 3 & $01 / 04 / 2014$ & $31 / 05 / 2014$ & 313 & 0.001 & 1.38 & 99 \\
\hline
\end{tabular}

*Primary cluster

$R R$ relative risk, $L L R$ Log likelihood ratio

given that the country has joined other Southern African countries to achieve malaria elimination [13, 73]. This will be achieved through alliances such as Elimination8 (E8) comprising Angola, Botswana, Mozambique, Namibia, South Africa Swaziland, Zambia and Zimbabwe. Closer collaboration in malaria elimination could be achieved through the ZAMZIM (Zambia and Zimbabwe), and the MOZAZI (Mozambique, Zambia and Zimbabwe), and the MOZIZA (Mozambique, Zimbabwe and South Africa) initiatives [3, 13, 73].

Nevertheless, insights generated in this study are useful in guiding further research on tightening cross border migration to malaria transmission and strengthening collaboration among neighbouring countries in the control of malaria. This is because without collaboration, malaria elimination is bound to fail as malaria occurrence due the influence of imported cases. Another potential limitation of the study is that although Kulldorff's scan statistic has been successfully used to detect circular clusters, it does not have the same success rate when detecting irregular clusters [75]. Despite these potential limitations, the results of this study are still important and may be useful for planning disease surveillance, particularly in areas of limited resources by focussing on high risk areas.

\section{Conclusions}

This study explored whether there is spatial heterogeneity in the distribution of malaria, one of the diseases of global public health concern. This was achieved through the detection of spatial and space-time clusters using scan statistics. The results indicated that high risk areas for malaria are concentrated in the northern, eastern, and south-eastern part of the country. The results of this study could be used to design malaria control strategies aimed at reducing malaria incidence in high risk areas particularly those along border areas. In addition, the results could be used to guide optimal resource allocation by giving priority to the regions in greatest need. The results of this study highlight the spatial heterogeneity in malaria occurrence with several high-risk areas detected across the country. Based on this retrospective study, significant attention need to be directed to high risk areas as these may act as reservoirs for the current and future malaria occurrence. The study is helpful in prioritizing resource allocation in high-risk areas for effective disease control. Although results are based on historical data, they are useful in tracking progress the country has made in reducing malaria incidence. In addition, the results can be used as a baseline to evaluate the impacts of malaria programmes implemented during this period which is important in informing current and future control strategies.

\section{Abbreviations}

DHIS: District Health Information System; GIS: Geographic Information System; IRS: Indoor residual spraying; MOZAZI: Mozambique, Zambia and Zimbabwe; MOZIZA: Mozambique, Zimbabwe and South Africa; SDG: Sustainable Development Goal; WHO: World Health Organisation; ZAMZIM: Zambia and Zimbabwe; ZIMSTAT: Zimbabwe National Statistics.

\section{Acknowledgements}

The authors would like to thank the government of Zimbabwe through the Ministry of Health and Child Care, Malaria Control Program for approving accessing to data and other valuable documents used in the write-up of this manuscript.

\section{Authors' contributions}

IG conceptualised the study and did the manuscript write-up. MM and GM analysed the data and presented the results. MDS was involved in manuscript integration. JC provided the epidemiological significance of the study while JM facilitated access to data used in this study. All authors read and approved the final manuscript.

\section{Authors' information}

IG is a senior lecturer in the Department of Geography Geospatial Sciences and Earth Observation at the University of Zimbabwe and specialises in geospatial health i.e., application of GIS and remote sensing to understand human and animal diseases in space. He currently leads the Geospatial research group in the department. MDS is a senior lecturer in the Geography Geospatial Sciences and Earth Observation at the University of Zimbabwe specialising in the application of GIS and remote sensing to various fields including health. MM and GM are graduate students from the University of Zimbabwe with specialisations in geospatial health. JC is a lecturer in the College of Health Sciences and has special interest in community medicine and infectious diseases. JM is the current director of the National Malaria Control Program in the Ministry of Health and Child Care, Zimbabwe.

\section{Funding}

No funding was received for this research.

\section{Availability of data and material}

The statistical methods presented in this manuscript were implemented in a freely downloadable software SaTScan version 9.4.2 available at (https://www. satscan.org/). The data used and/or analysed during the current study are available from the corresponding author on reasonable request. 
Ethics approval and consent to participate

Although human data was used in this study, it was only the aggregate total not individual subjects therefore "Not applicable".

\section{Consent for Publication}

Not applicable.

\section{Competing interests}

The authors declare that they have no competing interests.

\section{Author details}

${ }^{1}$ Department of Geography Geospatial Sciences and Earth Observation, University of Zimbabwe, P. O. Box MP 167, Mount Pleasant, Harare, Zimbabwe. ${ }^{2}$ Department of Community Medicine, University of Zimbabwe, 3rd Floor New Health Sciences Building, College of Health Sciences, P O Box A178, Avondale, Harare, Zimbabwe. ${ }^{3}$ National Malaria Control Program, Ministry of Health and Child Care, 4th Floor, Kaguvi Building, Central Avenue (Between 4th and 5th Street), Harare, Zimbabwe.

Received: 11 May 2020 Accepted: 14 October 2020

Published online: 22 October 2020

\section{References}

1. Feng $X$, Levens J, Zhou XN. Protecting the gains of malaria elimination in China. Infect Dis of Poverty. 2020;9:43.

2. Gwitira I, Murwira A, Zengeya FM, Shekede MD. Application of GIS to predict malaria hotspots based on Anopheles arabiensis habitat suitability in Southern Africa. Int J Appl Earth Obs Geoinf. 2018;64:12-21.

3. Deng T, Huang Y, Yu S, Gu J, Huang C. Spatial-temporal clusters and risk factors of hand, foot, and mouth disease at the district level in Guangdong Province, China. PLoS ONE. 2013;8(2):e56943.

4. WHO. World malaria report 2019. Geneva: World Health Organisation; 2019.

5. Campillo A, Daily J, Gonzalez IJ. International survey to identify diagnostic needs to support malaria elimination: guiding the development of combination highly sensitive rapid diagnostic tests. Malar J. 2017;16:385.

6. Masendu HT, Hunt RH, Koekemoer LL, Brooke BD, Govere J, Coetzee M, et al. Spatial and temporal distributions and insecticide susceptibility of malaria vectors in Zimbabwe. Afr Entomol. 2005;13:25-34.

7. Taylor P, Mutambu SL. A review of the malaria situation in Zimbabwe with special reference to the period 1972-1981. Trans R Soc Trop Med Hyg. 1986:80:12-9.

8. Khagayi S, Desai M, Amek N, Were V, Onyango ED, Odero C, et al. Modelling the relationship between malaria prevalence as a measure of transmission and mortality across age groups. Malar J. 2019;18:247.

9. Mfueni E, Devleesschauwer B, Aguirre AR, Malderen CV, Brandt PT, Ogutu $B$, et al. True malaria prevalence in children under five: Bayesian estimation using data of malaria household surveys from three subSaharan countries. Malar J. 2018;17:65.

10. WHO. World malaria report 2016. Geneva: World Health Organisation; 2016.

11. Yakob L, Cameron M, Lines J. Combining indoor and outdoor methods for controlling malaria vectors: an ecological model of endectocidetreated livestock and insecticidal bed nets. Malar J. 2017;11:1-13.

12. Sande S, Zimba M, Chinwada P, Masendu HT, Mberikunshe J, Makuwaza A. A review of new challenges and prospects for malaria elimination in Mutare and Mutasa Districts. Zimbabwe Malar J. 2016;15:1.

13. Sande S, Zimba M, Mberikunashe J, Tangwena A, Chimusoro A. Progress towards malaria elimination in Zimbabwe with special reference to the period 2003-2015. Malar J. 2017; 16:295.

14. WHO. Global technical strategy for malaria 2015. Geneva: World Health Organization; 2015a.

15. Alegana VA, Okiro EA, Snow RW. Routine data for malaria morbidity estimation in Africa: challenges and prospects. BMC Med. 2020;18:121.

16. Mosha JF, Sturrock HJ, Greenwood BM, Sutherland CJ, Gadalla NB, Atwal S. Hot spot or not: a comparison of spatial statistical methods to predict prospective malaria infections. Malar J. 2014;13:53.
17. Ernst KC, Adoka SO, Kowuor DO, Wilson ML, John CC. Malaria hotspot areas in a highland Kenya site are consistent in epidemic and non-epidemic years and are associated with ecological factors. Malar J. 2006;5:78.

18. Hundessa SH, Williams G, Li S, Guo J, Chen L, Zhang W, et al. Spatial and space-time distribution of Plasmodiumvivax and Plasmodiumfalciparum malaria in China, 2005-2014. Malar J. 2016;15:1-11.

19. Landier J, Rebaudet S, Piarroux R, Gaudart J. Spatiotemporal analysis of malaria for new sustainable control strategies. BMC Med. 2018;16:226.

20. Bousema T, Kreuels B, Gosling R. Adjusting for heterogeneity of malaria transmission in longitudinal studies. J Infect Dis. 2011;204:1-3.

21. Stresman GH. Beyond temperature and precipitation: ecological risk factors that modify malaria transmission. Acta Trop. 2010;116:167-72.

22. Mclafferty S. Disease cluster detection methods: recent developments and Public health implications. Ann GIS. 2015;21:127-33.

23. Hasyim H, Nursafingi A, Haque U, Montag D, Groneberg DA, Dhimal M, et al. Spatial modelling of malaria cases associated with environmental factors in South Sumatra, Indonesia. Malar J. 2018;17:87.

24. Carter R, Mendis KN, Roberts D. Spatial targeting of interventions against malaria. Bull World Health Organ. 2000;78:1401-11.

25. Magalhaes RJS, Langa A, Sousa-Figueiredo JC, Clements ACA, Nery SV. Finding malaria hot-spots in northern Angola: the role of individual, household and environmental factors within a meso-endemic area. Malar J. 2012;11:385.

26. Coleman M, Coleman M, Mabuza AM, Kok G, Coetzee M, Durrheim DN. Using the SaTScan method to detect local malaria clusters for guiding malaria control programmes. Malar J. 2009;8:68.

27. Loha E, Lunde TM, Lindtjørn B. Effect of bednets and indoor residual spraying on spatio-temporal clustering of malaria in a village in South Ethiopia: a longitudinal study. PLoS ONE. 2012;7(10):e47354.

28. Gaudart J, Poudiougou B, Dicko A, Ranque S, Toure O, Sagara I, et al. Space-time clustering of childhood malaria at the household level: a dynamic cohort in a Mali village. BMC Publ Health. 2006;6:286.

29. Wen L, Li C, Lin M, Yuan Z, Huo D, Li S, et al. Spatio-temporal analysis of malaria incidence at the village level in a malaria-endemic area in Hainan, China. Malar J. 2011;10:1-7.

30. Alemu K, Worku A, Berhane Y. Malaria infection has spatial, temporal and spatiotemporal heterogeneity in unstable malaria transmission areas in Northwest Ethiopia. PLoS ONE. 2013:8(11):e79966.

31. Manyangadze T, Chimbari MJ, Macherera M, Mukaratirwa S. Micro-spatial distribution of malaria cases and control strategies at ward level in Gwanda district, Matabeleland South, Zimbabwe. Malar J. 2017;16:476.

32. Xia J, Cai S, Zhang H, Lin W, Fan Y, Qiu J, et al. Spatial, temporal and spatiotemporal analysis of malaria in Hubei Province, China from 2004-2011. Malar J. 2015;14:145.

33. Gwitira I, Murwira A, Mberikunashe J, Masocha M. Spatial overlaps in the distribution of HIV/AIDS and malaria in Zimbabwe. BMC Infect Dis. 2018:18:1.

34. Wheeler DC. A comparison of spatial clustering and cluster detection techniques for childhood leukaemia incidence in Ohio, 1996-2003. Int J Health Geogr. 2007;6:13

35. Yamada I, Rogerson PA, Lee G. GeoSurveillance: a GIS-based system for the detection and monitoring of spatial clusters. J Geogr Syst. 2009;11:155-73.

36. Robertson C, Nelson TA. Review of software for space-time disease surveillance. Int J Health Geogr. 2010;9:16.

37. Wand $\mathrm{H}$, Ramjee $\mathrm{G}$. Targeting the hotspots: investigating spatial and demographic variations in HIV infection in small communities in South Africa. J Int AIDS Soc. 2010;13:41.

38. Tango T, Takahashi K. A flexible spatial scan statistic with a restricted likelihood ratio for detecting disease clusters. Stat Med. 2012;31:4207-18.

39. Barro AS, Kracalik IT, Malania L, Tsertsvadze N, Manvelyan J, Imnadze P, et al. Identifying hotspots of human anthrax transmission using three local clustering techniques. Appl Geogr. 2015;60:29-36.

40. Song C, Kulldorff M. Power evaluation of disease clustering tests. Int J Health Geogr. 2003;2:9.

41. Huang L, Pickle LW, Das B. Evaluating spatial methods for investigating global clustering and cluster detection of cancer cases. Stat Med. 2008;27:5111-42

42. Kulldorff M. A spatial scan statistic. Commun Stat. 1997;26:1481-96.

43. Ndhlovu F, Ndhlovu DN, Chikerema SM, Masocha M, Nyagura M, Pfukenyi DM. Spatio-temporal patterns of clinical bovine dermatophilosis in 
Zimbabwe 1995-2014. Onderstepoort J Vet Res. 2017. https://doi. org/10.4102/ojvr.v4184i4101.1386.

44. Mabaso MLH, Vounatsou MP, Smith T. Towards empirical description of malaria seasonality in southern Africa: the example of Zimbabwe. Trop Med Int Health. 2005;10:909-18.

45. Dehnavieh R, Haghdoost A, Khosravi A, Hoseinabadi F, Rahimi H, Poursheikhali A, et al. The District Health Information System (DHIS2): a literature review and metasynthesis of its strengths and operational challenges based on the experiences of 11 countries. Health Inf Manage J. 2018;48:62-75.

46. ESRI. ArcGIS Desktop. Release 10.3. 2011, Environmental Systems Research Institute: Redlands CA.

47. USAID. President's malaria initiative Zimbabwe: Malaria operational plan FY 2017. Washington: USAID 2016.

48. Rowe A, Kachur SP, Yoon SS, Lnych M, Sluster L, Steketee RW. Caution is required when using health facility-based data to evaluate the health impact of malaria control efforts in Africa. Malar J. 2009;8:209.

49. Gerrets R. Charting the road to eradication: Health facility data and malaria indicator generation in rural Tanzania. In: Rottenburg R, editor. The world of indicators: the making of governmental knowledge through quantification. Cambridge: Cambridge University Press; 2015. p. 36.

50. WHO. Global technical strategy for malaria elimination 2016-2030. Geneva: World Health Organization; 2015b.

51. ZIMSTAT. Zimbabwe Population Census 2012. Harare: ZIMSTAT 2012.

52. CSO. Zimbabwe National Population Census 2002. Harare: CSO 2002.

53. ZIMSTAT. Zimbabwe Population Projections Thematic Report. Harare: ZIMSTAT 2015.

54. Pfeiffer DU, Robinson TP, Stevenson M, Stevens KB, Rogers DJ, Clements AAC. Spatial analysis in epidemiology. New York: Oxford University Press; 2008

55. Poh-Chin L, Fun-Mun So KWC. Spatial epidemiological approaches in disease mapping and analysis. New York: CRC Press; 2009.

56. Chowdhury Al, Abdullah AYM, Haider R, Alam A, Billah SM, Bari S, et al. Analyzing spatial and space-time clustering of facility-based deliveries in Bangladesh. Trop Med Health. 2019;47:44.

57. Cheung YTD, Spittal MJ, Williamson MK, Tung SJ, Pirkis J. Application of scan statistics to detect suicide clusters in Australia. PLOS ONE. 2013;8(1):e54168.

58. Kulldorff M. Commentary: geographical distribution of sporadic Creutzfeldt-Jakob disease in France. Int J Epidemiol. 2002;31:495-6.

59. Chen J, Roth RE, Naito AT, Lengerich EJ, MacEachren AM. Geovisual analytics to enhance spatial scan statistic interpretation: an analysis of US Cervical cancer mortality. Int J Health Geogr. 2008;7:57.

60. Zhang W, Wang L, Fang L, Ma J, Xu Y, Jiang J, et al. Spatial analysis of malaria in Anhui province, China. Malar J. 2008;7:206.

61. Liu Y, Wang X, Liu Y, Sun D, Ding S, Zhang B, et al. Detecting spatial-temporal clusters of HFMD from 2007 to 2011 in Shandong Province, China. PLoS ONE. 2013;8(5):e63447.

62. Gwitira I, Murwira A, Masocha M, Zengeya FM, Shekede MD, Chirenda J, et al. GIS-based stratification of malaria risk zones for Zimbabwe. Geocarto Int. 2019:34:1163-76.
63. Bannister-Tyrrell M, Verdonck K, Hausmann-Muela S, Gryseels C, Ribera $J M$, Grietens KP. Defining micro-epidemiology for malaria elimination: systematic review and meta-analysis. Malar J. 2017;16:164.

64. UNDESA. Sustainable Development Goal 3: Ensuring Health Lives and Promote Well-Being for All at All Ages. 2015: https://sustainabledeve lopment.un.org/sdg3. Accessed on 15 September 2019.

65. Okello G, Molyneux S, Zakayo S, Gerrets R, Jones C. Producing routine malaria data: an exploration of the micro-practices and processes shaping routine malaria data quality in frontline health facilities in Kenya. Malar J. 2019;18:420.

66. Byass P. Making sense of long-term changes in malaria. Lancet. 2008;372:1523-5.

67. Manyangadze T, Chimbari MJ, Gebreslasie M, Mukaratirwa S. Risk factors and micro-geographical heterogeneity of Schistosoma haematobium in Ndumo area, uMkhanyakude district, KwaZulu-Natal. South Africa Acta Tropica. 2016;159:176-84.

68. Saita S, Silawan T, Parker DM, Sriwichai P, Phuanukoonnon S, Sudathip P, et al. Spatial heterogeneity and temporal trends in malaria on the ThaiMyanmar Border (2012-2017): a retrospective observational study. Trop Med Infect Dis. 2019;4:62

69. Xu X, Zhou G, Wang Y, Hu Y, Ruan Y, Fan Q, et al. Microgeographic heterogeneity of border malaria during elimination phase, Yunnan Province, China, 2011-2013. Emerg Infect Dis. 2016;22:8.

70. Prashanthi DM, Manickiam B, Balasubramanian S. Use of of Remote Sensing and GIS for monitoring the Environmental factors associated with Vector-borne Disease (Malaria). Third International Conference on Environment and Health. Chennai: Department of Geography. University of Madras and Faculty of Environmental Studies 2003.

71. Muchena G, Dube B, Chikodzore R, Pasipamire J, Murugasampillay S, Mberikunashe $J$. A review of progress towards sub-national malaria elimination in Matabeleland South Province, Zimbabwe (2011-2015): a qualitative study. Malar J. 2018;17:146.

72. Adeola A, Ncongwane K, Abiodun G, Makgoale T, Rautenbach H, Botai J, et al. Rainfall trends and malaria occurrences in Limpopo Province, South Africa. Int J Environ Res Publ Health. 2019;16:5156.

73. SADC. SADC Malaria Elimination Eight Initiative: Annual Report 2019. Windhoek: Elimination8 2019.

74. Kamuliwo M, Chanda E, Haque U, Mwanza-Ingwe M, Sikaala C, KatebeSakala C, et al. The changing burden of malaria and association with vector control interventions in Zambia using district-level surveillance data, 2006-2011. Malar J. 2013;12:437.

75. Aamodt G, Samuelsen SO, Skrondal A. A simulation study of three methods for detecting disease clusters. Int J Health Geogr. 2006;5:15.

\section{Publisher's Note}

Springer Nature remains neutral with regard to jurisdictional claims in published maps and institutional affiliations.

\footnotetext{
Ready to submit your research? Choose BMC and benefit from:

- fast, convenient online submission

- thorough peer review by experienced researchers in your field

- rapid publication on acceptance

- support for research data, including large and complex data types

- gold Open Access which fosters wider collaboration and increased citations

- maximum visibility for your research: over $100 \mathrm{M}$ website views per year
}

At BMC, research is always in progress.

Learn more biomedcentral.com/submissions 